\title{
Persistent DNA Contamination in Competitive RT-PCR Using cRNA Internal Standards: Identity, Quantity, and Control
}

BioTechniques 32:1412-1417 (June 2002)

\author{
Jennifer L.K. Matthews, May \\ Chung, and John Robert \\ Matyas \\ University of Calgary Faculty \\ of Medicine, Calgary, Alberta, \\ Canada
}

\section{INTRODUCTION}

RT-PCR is a powerful procedure for detecting specific mRNAs, yet quantifying mRNA copy number remains challenging. A complementary RNA (cRNA) standard can be introduced at the RT step to quantify target mRNA copy number $(8,9)$. Such cRNA “internal" standards share PCR primer sequences with the mRNA target and can differ in size to distinguish the PCR products of cRNA standard from target mRNA. In this "competitive RT-PCR", the quality of target RNA and cRNA determines the accuracy of estimating mRNA copy number. RNA extracted from tissues is often treated to eradicate genomic DNA $(2,12,15)$, but much less attention has been given to template DNA contamination in the cRNA products of in vitro transcription. Commercial kits often prescribe digestion with RNase-free DNase I after in vitro transcription, though few describe the assessment of residual DNA contamination in a "No-RT" PCR (no reverse transcriptase enzyme added). While the No-RT PCR controls of target mRNA have been consistently blank in our experiments, the No-RT PCR controls of cRNA internal standard have been persistently positive, indicating residual contamination with plasmid-template DNA. We explored this problem further because any residual DNA contamination, if unrecognized, can lead to the overestimation of mRNA copy number. Thus, we sought to rid our cRNA internal standards of DNA contamination.

\section{MATERIALS AND METHODS}

Canine ligament was obtained at necropsy, snap-frozen, pulverized in a Micro Dismembrator (B. Braun Biotech. Allentown, PA, USA) cooled in liquid nitrogen. Total RNA was extracted by the TriSpin method (15). Briefly, $0.3 \mathrm{~mL}$ chloroform were added to pow dered tissue already suspended in $1 \mathrm{~mL}$ TRIzOL ${ }^{\circledR}$ reagent (Invitrogen, Carlsbad, CA, USA). After centrifuging at $13400 \times g$ for $5 \mathrm{~min}$ at $4^{\circ} \mathrm{C}$, the supernatant was loaded on an RNeasy ${ }^{\circledR}$ column (Qiagen, Valencia, CA, USA) and total RNA was eluded with $30 \mu \mathrm{L}$ RNase-free water. Tissue RNA and internal standard cRNA were quantified using SYBR Green ${ }^{\circledR}$ (Molecular Probes, Eugene, OR, USA) spectrofluorometry; the integrity of these RNAs was assessed visually on an RNA gel.

Type I procollagen internal standard cRNA was transcribed in vitro using 1.5-2.0 $\mu \mathrm{g}$ plasmid DNA template using the MEGAScript ${ }^{\mathrm{TM}}$ High Yield Transcription Kit (Ambion, Austin, TX, USA). The in vitro transcription $(20 \mu \mathrm{L})$ was done at $37^{\circ} \mathrm{C}$ for up to $8 \mathrm{~h}$. If included, $2 \mu \mathrm{L}$ RNase-free DNase I (Am bion) was added to the in vitro transcription reaction, incubated for $15 \mathrm{~min}$ at $37^{\circ} \mathrm{C}$, and terminated with $1.5 \mu \mathrm{L} 0.2 \mathrm{M}$ (1.33 mM final concentration) EDTA. cRNA was precipitated with $7 \mu \mathrm{L} 4 \mathrm{M}$ (0.124 $\mathrm{M}$ final concentration) lithium chloride and $225 \mu \mathrm{L} 100 \%$ ethanol for $45 \mathrm{~min}$ at $-80^{\circ} \mathrm{C}$. The cRNA pellet was obtained by centrifugation, followed by two washes with $225 \mu \mathrm{L} 70 \%$ ethanol, and was dried under vacuum before the treatments outlined in Table 1.

Table 2 lists the forward and reverse PCR primer sequences for native type I collagen. Thermal cycling parameters were $1 \mathrm{~min}$ at $94^{\circ} \mathrm{C}, 2 \mathrm{~min}$ at $54^{\circ} \mathrm{C}$, and $3 \mathrm{~min}$ at $72^{\circ} \mathrm{C}$ for 30 cycles. Fifteen microliters of cDNA was used in each 50- 
Table 1. Strategies of Reducing Template DNA Contamination

\begin{tabular}{|c|c|c|c|c|}
\hline Strategy & $\begin{array}{l}\text { DNA Removal } \\
\text { Method }\end{array}$ & $\begin{array}{c}\text { Template DNA } \\
\text { Used for } \\
\text { In Vitro } \\
\text { Transcription }\end{array}$ & $\begin{array}{c}\text { DNase I } \\
\text { Treatmenta }\end{array}$ & $\begin{array}{c}\text { Percent } \\
\text { Contaminationb } \\
\text { *DNA* / (IS-DNA } \\
\text { - }{ }^{*} \text { DNA }^{*} \text { ) } \text { × } 100\end{array}$ \\
\hline I.A & \multirow[t]{2}{*}{ Digestion } & Full-length template & $\checkmark$ & $2.16 \%$ \\
\hline I.B & & Template fragment & $\checkmark$ & $0.20 \%$ \\
\hline II.A & \multirow{6}{*}{ Extraction } & Template fragment & $\checkmark$ & $1.39 \%$ \\
\hline II.B & & Template fragment & - & $1.43 \%$ \\
\hline II.C & & Full-length template & $\checkmark$ & $3.58 \%$ \\
\hline II.D & & Full-length template & - & $0.14 \%$ \\
\hline \multirow[t]{2}{*}{ II.E } & & $\begin{array}{l}\text { Full-length template } \\
\text { end-labeled with }\end{array}$ & & \\
\hline & & digoxigenin & 一 & $-c$ \\
\hline III & Separation & Full-length template & $\checkmark$ & $0.96 \%$ \\
\hline \multicolumn{5}{|c|}{$\begin{array}{l}\text { aAmbion RNase-free DNase I with the recommended amount of } 2 \mathrm{U} / \mu \mathrm{g} \text { DNA and } \\
15-\text { min incubation at } 37^{\circ} \mathrm{C} \\
\mathrm{b}^{*} \mathrm{DNA}{ }^{*} \text { signifies template DNA contamination. } \\
\text { cNo cRNA was recovered after end-labeling and in vitro transcription. }\end{array}$} \\
\hline
\end{tabular}

Table 2. Canine-Specific Type I Procollagen Primer Sequences for Standard PCR and the Mutagenic Primers Designed to Create the Internal Standard Size-Mutant cDNA

\begin{tabular}{|llcl|}
\hline Primers & \multicolumn{1}{c}{ Sequence } & Size (bp) & Source \\
\hline COLI-F & 5'-GATGCGTTCCAGTTCGAGTA-3' & 312 & Kao, Wo \\
COLI-R & 5'-GGTCTTCCGGTGGTCTTGTA-3' & & Kao, Wb \\
COLI-R,M & 5'-GGTCTTCCGGTGGTCTTGTATA- & 248 & $\mathrm{C}$ \\
& GGTGAAGCGCTGTTGCCC-3' & & \\
aF, forward, sense primer; R, reverse, antisense primer; R,M, mutagenic primer \\
$\begin{array}{l}\text { derived from the original reverse primer. This primer is used with the forward } \\
\text { primer to create the size-mutant internal standard for collagen type I. } \\
\text { bUnpublished data } \\
\text { cThe method of Celi et al. (5) was used to design this primer. }\end{array}$ \\
\hline
\end{tabular}

$\mu \mathrm{L}$ PCR. The remaining $35-\mu \mathrm{L}$ PCR volume contained $5 \mu \mathrm{L} 10 \times$ PCR buffer (Invitrogen), $0.2 \mathrm{mM}$ dNTPs, $0.5 \mu \mathrm{M}$ each forward and reverse primers, $23.75 \mu \mathrm{L}$ molecular-grade water, and $0.25 \mu \mathrm{L}$ Taq DNA polymerase (Amersham Biosciences, Baie d'Urfé, Quebéc, Canada). PCR products were visualized on $1 \%$ agarose gels stained with ethidium bromide.

The size-mutant internal standard sequence of type I procollagen mRNA was generated by PCR mutagenesis (5). The mutagenic primer (Table 2) skipped $64 \mathrm{bp}$ while preserving the reverse primer site. The size-truncated cDNA was cloned into SK(+)-PCR Script (AMP Cloning Kit ${ }^{\mathrm{TM}}$; Stratagene, La Jolla, CA, USA). Plasmid DNA was purified with the QIAprep ${ }^{\circledR}$ Spin Miniprep
Columns (Qiagen). Plasmid DNA was linearized with Pst-1 (Amersham Biosciences) and quantified by optical densitometry before in vitro transcription.

For No-RT PCR, $1 \mu \mathrm{g}$ internal standard cRNA or sample total RNA was combined with all the reaction components of the reverse transcription kitexcept the reverse transcriptase enzyme-to a final volume of $500 \mu \mathrm{L}$ (ProSTAR $^{\mathrm{TM}}$ First-Strand RT-PCR Kit; Stratagene). In a typical competitive PCR experiment to assess type I collagen mRNA in ligament, the internal standard cDNA was diluted $10^{3}$ - to $10^{12}$-fold before amplification. To assess DNA contamination, No-RT PCR was done without dilution ( $10^{0}$ times $)$, then at dilutions of $10^{2}, 10^{4}, 10^{6}$, and $10^{8}$ times. A negative control without nucleic acid and positive controls with RT product of type I procollagen internal standard cDNA were included in each experiment.

Initially, No-RT contamination was studied by dilution, which was only qualitative. As band intensities were not proportionate to the dilutions, it was difficult to extrapolate meaningful values of contaminant copy number; for this, the "inverted" competition was developed (Figure 1). Type I collagen cDNA was amplified by RT-PCR from ligament RNA using the primers listed in Table 1 and isolated from an $0.8 \%$ agarose gel (QIAquick ${ }^{\circledR}$ Mini-Elude Gel Extraction Columns; Qiagen). The purified cDNA was quantified using PICOGreen ${ }^{\circledR}$ (Molecular Probes). Dilutions of native cDNA were mixed with constant amounts of No-RT reaction products (contaminating template DNA) in a competitive series (Figure 1). For each No-RT sample, the corresponding RT sample was also run in a separate competitive PCR experiment to quantify the internal standard cDNA plus contaminant DNA. All purification strategies were assessed by this method (Table 1).

A constant amount of No-RT or RT sample was used in competitive PCR with varying, known quantities of nativesized cDNA. Eight competitive reactions contained between $1 \times 10^{-1}$ and $1 \times 10^{-8}$ ng native $\mathrm{cDNA}$. Twenty-five microliters of each $50 \mu \mathrm{L}$ PCR reaction were run on a $2 \%$ agarose gel, stained with ethidium bromide, and photographed on Polaroid ${ }^{\circledR}$ Proplan 55 film. The integrated absorbance of bands on the photonegative was evaluated by 2-D scanning densitometry (Scantalytics; Emerston Instruments, Richmond Hill, Ontario, Canada). Once corrected for differences in the molecular size, the copy number of unknown molecules was calculated from the log of the ratio of the integrated density of known template to unknown contaminant or cDNA (9).

\section{RESULTS}

Three basic strategies were tested for their effectiveness to minimize DNA contamination in cRNA standards: $(i)$ DNase digestion, (ii) selective DNA extraction, and (iii) size separation. Table 1 lists eight different experiments em 
ploying one or more of these strategies for DNA removal. After in vitro transcription and cRNA purification, com petitive PCR was performed using known amounts of (full-length) native cDNA and the products of either RT (size-reduced cDNA contaminated with unknown amounts of template DNA) or No-RT (template DNA contamination, if any) reactions (Figures 1 and 2). Since this is opposite to our usual practice, we termed this an inverted competition. The percentage of DNA contamination was calculated as the number of contaminants (determined by No-RT competitive PCR), divided by the total copy number determined in competitive RT-PCR (internal standard cDNA plus contaminants) times 100 (Table 1). Negative blank controls (without DNA) were included to rule out systematic DNA contamination; positive controls for specific PCR products were also included in every experiment.

\section{Strategy I: DNase Digestion}

Under the recommended conditions, we found that digestion of DNA ladder was incomplete with all DNase products (Sigma, Roche Applied Science, Qiagen, and Ambion); with longer digestion times and increased enzyme concentrations, DNA ladder was com pletely digested, yet with the exception of Ambion's RNase-free DNase I, the integrity of cRNA from in vitro transcription reactions was compromised (data not shown) and was still contaminated with DNA (Table 1). Ambion's DNase was used for the remaining experiments requiring DNase digestion. Reducing DNA in the in vitro transcription from approximately $3.2 \mathrm{~kb}$ (the plasmid plus the internal standard tem plate) to $413 \mathrm{bp}$ (the internal standard template plus some flanking sequences) reduces but does not eliminate cDNA contamination (Table 1, Strategy IB).

\section{Strategy II: Selective Extraction of DNA from cRNA}

Selective partitioning of DNA into acidified phenol (6) failed to eliminate DNA contamination in in vitro transcription regardless of $(i)$ the size of the starting DNA template, (ii) DNase treatment, or (iii) sterol (digoxigenin) modification of the DNA template (Table 1). The integrity of cRNA isolated by this method was excellent.

\section{Strategy III: Size Separation}

High-quality cRNA visibly separated from plasmid-template DNA in both agarose (formaldehyde) and polyacrylamide (urea) gels, but only cRNA bands from polyacrylamide gels could be successfully reverse-transcribed. Gel purification protocols that include high-temperature treatments to separate cRNA-template DNA hybrids did not completely eliminate DNA contamination (Table 1, Strategy III).

\section{DISCUSSION}

PCR is an extremely sensitive method for analyzing samples that contain specific sequences of DNA. Therefore, to ensure the accurate quantification of mRNA by competitive PCR, an ideal cRNA internal standard would be free of template DNA before reverse transcription. Yet, despite rigorous treatment using a number of different strategies, we discovered persistent DNA contamination in the cRNA preparations after in vitro transcription.

This DNA contamination of cRNA internal standards is rarely mentioned. A search for "competitive RT-PCR" in PubMed returned some 1300 papers since 1992. A systematic sample of 100 papers found that nearly a third (28) used cDNA "mimics" (i.e., no control of $\mathrm{RT}$ ); of those 72 papers using RNA standards, 50 made no mention of purifying the cRNA. Of those 22 papers that did, 21 used RNase-free DNase I treatment of in vitro transcription products before competitive PCR (e.g., References 7, 10,11 , and 13), and two used gel purification $(4,17)$. Only eight papers reported No-RT controls (e.g., Reference 11), though it was unclear exactly how the No-RT PCR was conducted and if these samples were diluted before PCR. Only one paper (14) reported persistent DNA contamination of their cRNA standards. These authors also assessed No-RT samples in a dilution series $\left(10^{0}, 10^{2}\right.$, and $10^{4}$ times, etc.), and discovered, as did we, that DNA contamination is undetectable in PCR at dilutions greater than about $10^{4}$ times. We suspect that some authors diluted their No-RT controls along with their experimental RT samples (containing their cRNA standards), typically from $10^{3}$ to $10^{12}$ times,

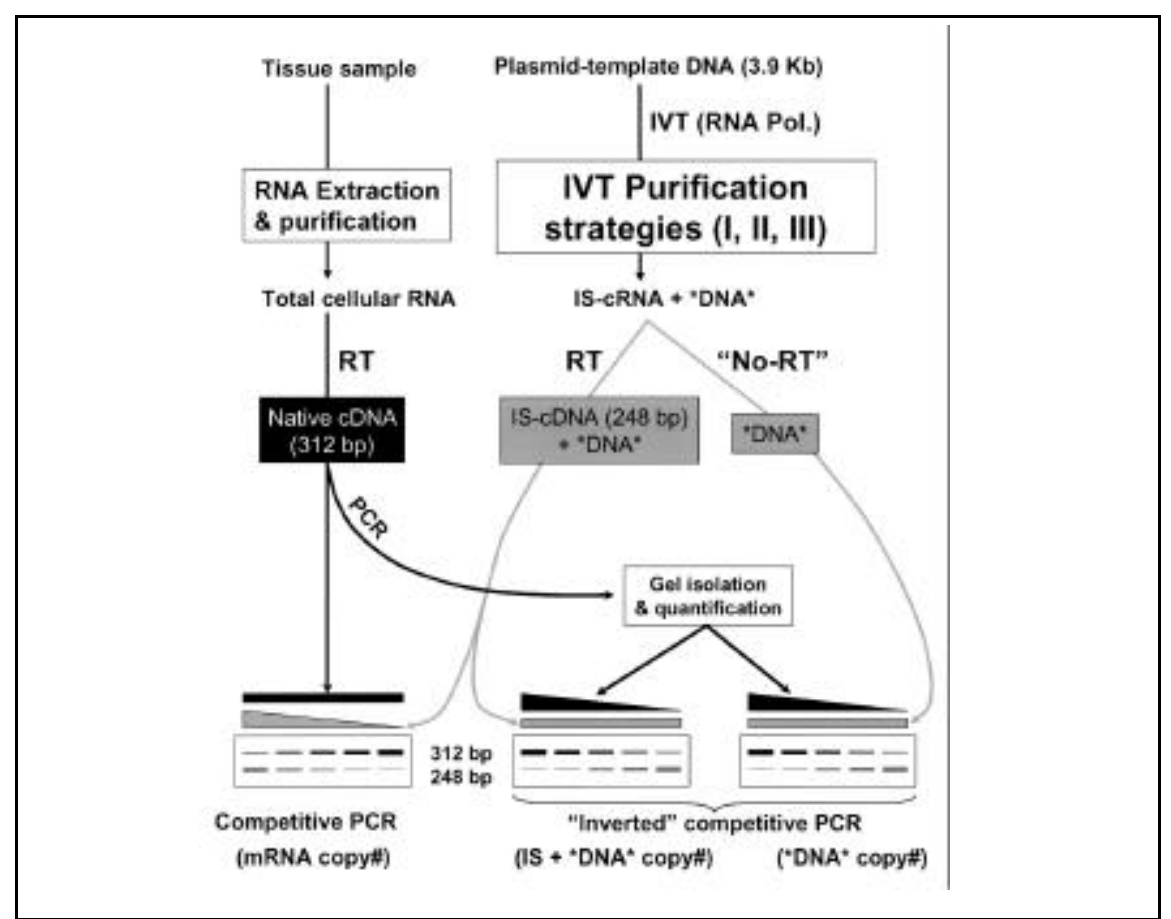

Figure 1. Diagram of experimental design. IVT, in vitro transcription; IS, internal standard; contaminating template DNA is signified as *DNA*. 


\section{Research Report}

thereby making the relatively low num ber of contaminants undetectable by PCR (e.g., References 9, 10, 16, 18, and 19). It is noteworthy that the percentage of template DNA contamination in the internal standard remains the same re-

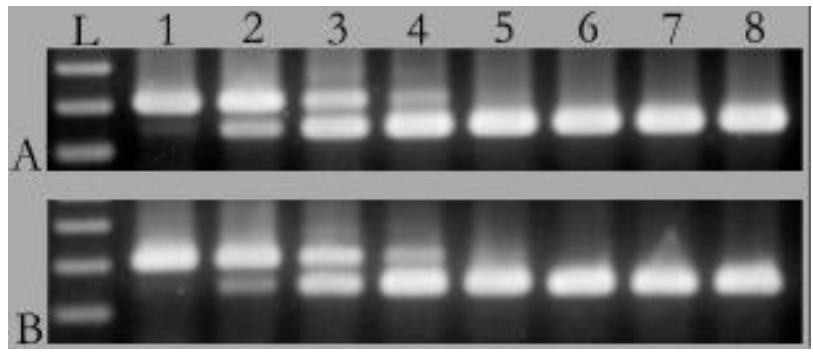

Figure 2. An inverted competitive PCR to quantify DNA contamination in internal standard-cRNA using strategy II.A (Table 1). A known amount of native cDNA was added at various dilutions to a constant volume of either RT (A) or No-RT (B) samples. PCR products of native template (312 bp); the contaminant DNA and internal standard cDNA products are $248 \mathrm{bp}$. Lanes 1-8 dilutions of native cDNA (ng); lane L, $1 \mathrm{~Kb}$ Plus DNA ladder ${ }^{\mathrm{TM}}$ (Invitrogen); lane $1,1 \times 10^{-1}$; lane $2,1 \times 10^{-2}$; lane $3,1 \times 10^{-3}$; lane $4,1 \times 10^{-4}$; lane $5,1 \times 10^{-5}$; lane $6,1 \times 10^{-6}$; lane $7,1 \times 10^{-7}$; lane $8,1 \times 10^{-8}$. dards is relatively minor, which suggests that routine RNase-free DNase I digestion is nearly complete. DNA contamination notwithstanding, there seems to be a trade-off between the completeness of DNA digestion and the preservation of cRNA integrity. It is unclear exactly what disturbs the integrity of cRNA during digestion, though RNase contamination and the method of DNase I inactivation have been suggested as possible explanations (1,2). Degradation of cRNA is as much a concern as DNA contamination since the amount of cRNA added as a competitor is typically assessed by optical densitometry or fluorometry, methods that cannot distinguish between incomplete and complete cRNA sequences. Should degradation of cRNA involve the PCR primer sites, competitive PCR will overestimate the true number of target mRNAs.

We sought an alternative to DNase digestion for purifying cRNA stan- 
dards; however, there were many challenges. Size-fractionation of DNA template from cRNA product seemed an ideal solution to the problem. However, the purification of RNA from gels was difficult: it seems likely that traces of formaldehyde inhibit the reverse transcriptase enzyme when cRNA is isolated from agarose gels. While cRNA isolation and RT were successful after urea-polyacrylamide gel electrophoresis, we still found some template DNA contamination. Supplementary experiments proved that small amounts of contaminating DNA escape into the gel running buffer and are picked up when cutting out cRNA bands from the gel. Similarly, attempts to selectively extract DNA with the TRIzOL reagent (containing acidified phenol) were incomplete (Table 1 ). While $1 \%$ contamination does not seem threatening, it is sobering to calculate that, for our type I collagen internal standard, this translates into $3.7 \times 10^{10}$ copies of $\mathrm{DNA} / \mu \mathrm{L}$ undiluted cRNA standard. This contaminant seems worth recognizing as a potential contributor to experimental error. Though not significantly different from the other purification strategies, we routinely use TRIzOL because it is superior in preserving cRNA integrity.

We have used TRIzOL extensively to extract high-quality RNA from tissues without appreciable genomic DNA contamination (12) and yet regularly found template DNA contamination in our synthetic cRNA. This paradox is seemingly due to the number of specific primer sites found in these samples. Whereas few strands of genomic DNA share primer sites with our mRNAs, every strand of template DNA shares primers with the cRNA standard. In other words, No-RT PCR detects genomic DNA in total RNA only when the DNA shares the specific primers tested. We postulate that, on a molar basis, the amount of genomic DNA contamination is similar to that of tem plate DNA, which is more easily detected by PCR because of the higher number of specific primer sites in the products of in vitro transcription.

Standards are essential for many experiments, and the key to a useful standard is its full characterization. The cRNA standard used in competitive RT-PCR is no exception, and our in- ability to purify it completely is disheartening. Nonetheless, we have characterized our internal standard and its DNA contaminant; we have also developed a method for evaluating the contamination as percentage of total copies. For low levels of contamination, quantifying template cDNA contamination is a practical alternative to synthesizing RNA standards (the estimated com mercial cost is CDN\$3800 for one 248bp standard). The popularity of realtime thermal cyclers does not diminish the significance of the present findings: RNA standards are still needed to assess the efficiency of reverse transcription, and DNA contaminants can be invisible in these experiments.

\section{ACKNOWLEDGMENTS}

J.R.M. is a Senior Scholar of The Arthritis Society. J.R.M. also receives operating support from the Canadian Institutes for Health Research, the Natural Sciences and Engineering Research Council of Canada, and The Canadian Arthritis Network Centres of Excellence. The authors are grateful for the advice of Dr. Mayi Arcellana-Panlilio.

\section{REFERENCES}

1.Ambion. 2000. Tips from the bench: is your DNase RNase-free? Technotes 7(4).

2.Ambion. 1999. Tips from the bench: is Your RNA DNA-free? Technotes 6(5).

3.Ambion. 2000. Tips from the bench: the truth: your RNA is contaminated! A new method to remove DNA. Technotes 7(1).

4.Cao, Z., Y. Wang, E.A. Reid, G. McShepard, M. Kemp, R.F. Newkirk, and J.G. Townsel. 2001. The quantitative distribution of a putative PKC epsilon mRNA in Limulus central nervous system by modified competitive RT-PCR J. Neurosci. Methods 105:193-199.

5.Celi, S., M. Zenilman, and A. Shuldiner. 1993. A rapid and versatile method to synthesize internal standards for Ccompetitive PCR. Nucleic Acids Res. 21:1047.

6.Chomczynski, P. and N. Sacchi. 1987. Singlestep method of RNA isolation by acid guanidinium thiocyanate-phenol-chloroform extraction. Anal. Biochem. 162:156-159.

7.Evans, C.W., D.A. Wilson, and G.N. Mills. 2001. Quantitative competitive (qc) RT-PCR as a tool in biomarker analysis. Biomarkers 6:7-14.

8.Foley, K., M. Leonard, and J. Engel. 1993. Quantitation of RNA using the polymerase chain reaction. Trends Genet. 3:380-385.

9.Gilliland, G., S. Perrin, K. Blanchard, and H. Franklin Bunn. 1990. Analysis of cytokine mRNA and DNA: detection and quantitation by competitive polymerase chain reaction. Proc. Natl. Acad. Sci. USA 87:2725-2729.

10.Goerke, C., M. Bayer, and C. Wolz. 2001. Quantification of bacterial transcripts during infection using competitive reverse transcription-PCR (RT-PCR) and LightCycler RT-PCR Clin. Diagn. Lab. Immunol. 8:279-282.

11.Iascone, M.R., S. Vittorini, A. Collavoli, A. Cupelli, G. Kraft, A. Biagini, and A. Clerico. 1999. A rapid procedure for the quantitation of natriuretic peptide RNAs by competitive RTPCR in congenital heart defects. J. Endocrinol. Invest. 22:835-842.

12.Marchuk, L., P. Sciore, C. Reno, C.B. Frank, and D.A. Hart. 1998. Postmortem stability of total RNA isolated from rabbit ligament, tendon and cartilage. Biochim. Biophys. Acta 1370:171-177.

13.McGrath, J.S., G. Dooley, and R.W. Haylock. 2000. Quantification of Clostridium botulinum toxin gene expression by competitive reverse transcription-PCR. Appl. Environ. Microbiol. 66:1423-1428.

14.Pinti, M., J. Pedrazzi, F. Benatti, V. Sorrentino, C. Nuzzo, V. Cavazzuti, P. Biswas, D.N. Petrusca, et al. 1999. Differential down-regulation of CD95 or CD95L in chronically HIVinfected cells of monocytic or lymphocytic origin: cellular studies and molecular analysis by quantitative RT-PCR. FEBS Lett. 458:209-214.

15.Reno, C., L. Marchuk, P. Sciore, C.B. Frank, and D.A. Hart. 1997. Rapid isolation of total RNA from small samples of hypocellular, dense connective tissues. BioTechniques 22:1082-1086

16.Rots, M.G., J.C. Willey, G. Jansen, C.H. Van Zantwijk, P. Noordhuis, J.P. DeMuth, E. Kuiper, A.J.P. Veerman, et al. 2000. mRNA expression levels of methotrexate resistance-related proteins in childhood leukemia as determined by a standardized competitive templatebased RT-PCR method. Leukemia 14: 2166-2175.

17.Siegwart, J.T., Jr. and T.T. Norton. 2001. Steady state mRNA levels in tree shrew sclera with form-deprivation myopia and during recovery. Invest. Ophthalmol. Vis. Sci. 42:11531159.

18. Venturi, G., R. Ferruzzi, L. Romano, C. Marinunzia, P.E. Valensin, and M. Zazzi. 2000. Ultrasensitive in-house reverse transcription-competitive PCR for quantiation of HIV-1 RNA in plasma. J. Virol. Methods 87:91-97.

19.Wang, A.M., M.V. Doyle, and D.F. Mark. 1989. Quantitation of mRNA by the polymerase chain reaction. Proc. Natl. Acad. Sci. USA 86:9717-9721.

Received 29 October 2001; accepted 22 February 2002.

Address correspondence to:

Dr. John Robert Matyas

The McCaig Centre for Joint Injury and Arthritis Research

Departments of Cell Biology \& Anatomy and Pathology \& Laboratory Medicine

University of Calgary Faculty of Medicine 3330 Hospital Drive NW

Calgary, Alberta T2N 4N1, Canada

e-mail:jmatyas@ucalgary.ca 